installation also lagged behind the 1985 deadline and peaked following a series of EtO citations by OSHA. From 1990 through 1992, 23\% of hospitals reported having exceeded the action level once or more, $24 \%$ reported having exceeded the excursion limit, and 33\% reported that workers were exposed accidentally to EtO in the absence of personal monitoring.

The authors conclude that, almost a decade after the publication of the EtO standards, exposure-monitoring requirements were widely but incompletely implemented. Work-shift exposures had decreased markedly since the mid-1980s, but overexposure continued to occur widely. OSHA enforcement appears to have stimulated implementation, but additional exposure monitoring is needed. In addition to EtO being a known human carcinogen, it also is a potent allergic sensitizer, and the increased reports of EtO-associated asthma in healthcare workers further emphasizes the need to control worker exposures.

FROM: LaMontagne A, Kelsey K. Evaluating OSHA's ethylene oxide standard: employer exposure-monitoring activities in Massachusetts hospitals from 1985 through 1993. Am J Public Health 1997;87:1119-1125.

\section{Reliability of TB Skin-Test Measurement Using Ballpoint Pen}

The tuberculin skin test has many potential sources of error and variability. Standardization of the tuberculin reagent and interpretation of results have been considered in detail; however, little attention has been given to the reading itself. Measurement of the induration is one of the most important potential sources of error. Induration often is difficult to define with the use of the customary technique of palpation.

An alternative technique that has been advocated as early as 1975 never has been discussed in official statements on tuberculosis. Researchers from France and British Columbia recently investigated the reliability of the ballpoint pen technique and compared this technique with the palpation method. With the ballpoint pen technique, a medium ballpoint pen is used to draw a line starting 1 to 2 $\mathrm{cm}$ away from the skin reaction and moving toward its center. When the pen reaches the margin of the induration, an increased resistance to further movement is felt, and the pen is lifted. The procedure is repeated on the opposite side of the skin reaction. The distance between the ends of the opposing lines at the margins of the induration is measured.

Three measurements were taken on 69 persons with reactions to the TB skin test: two with the ballpoint pen technique and one with palpation. The results indicated that intra- and interobserver reliability coefficients of the ballpoint pen technique were high. Five percent of the time, however, a second measurement by the same observer could be at least $2.7 \mathrm{~mm}$ less to $3.0 \mathrm{~mm}$ more than the first measurement, and the measurement from the second observer could be at least $3.4 \mathrm{~mm}$ less to $3.7 \mathrm{~mm}$ more than the measurement from the first observer. This could lead to the reclassification of a positive test as negative or vice versa. The area of imprecision was 38\% less broad for the ballpoint pen technique than for the palpation technique.

The researchers concluded that the ballpoint pen technique is a reliable technique for measurements of TB skin test results. They suggest that current methods for TB skin testing reading should be reconsidered.

FROM: Pouchet J, Grasland A, Collet C, Coste J, Esdaile JM, Vinceneux P. Reliability of tuberculin skin test measurement. Ann Intern Med 1997;126:210-214.

\section{Prevention of Opportunistic Infections in HIV-Infected Persons- Revised Guidelines}

The US Public Health Service and the Infectious Disease Society of America issued revised "1997 Guidelines for the Prevention of Opportunistic Infections in Persons with HIV.” These guidelines update the guideline published in 1994. Among the most important changes are the recommendation that clarithromycin or azithromycin be considered first-choice drugs for Mycobacterium avium complex prophylaxis, with rifabutin as an alternative, and a revision in the immunization schedule for HIV-infected children, with a comparison of the differences between this schedule and that for immunocompetent children.

Although not included in the disease-specific recommendations, an important issue in opportunistic-infection prophylaxis is whether to offer or continue prophylaxis on the basis of the lowest CD4+ T-lymphocyte count or of a more recent count that has been elevated as a result of antiretroviral therapy. This issue is particularly pertinent because of the administration of potent drug combinations that include protease inhibitors, which may increase CD4+ counts by 100 to 250 cells per milliliter. It currently is unknown whether such increases in CD4+ counts provide anti-infective protection comparable with that afforded to patients whose counts never declined below the current level. Until data assessing these risks are available, most experts recommend that prophylaxis be initiated or continued on the basis of the lowest CD4+ count.

FROM: Centers for Disease Control and Prevention. 1997 USPHS/IDSA guideline for the prevention of opportunistic infections in persons with human immunodeficiency virus. $M M W R$ 1997;46(No. RR-12):1-46.

Additional news items in this issue: Lack of Isolation Despite Respirator Use Leads to MDR-TB Outbreak, page 709. 